\title{
QUAND UN SEUL ÊTRE VOUS MANQUE : REGARD FRANÇAIS SUR LA BIVALENCE CULTURELLE DE L'ÊTRE DANS LA LANGUE ESPAGNOLE À TRAVERS UNE PERCEPTION CERVANTESQUE DES VERBES «SER » ET «ESTAR »
}

\author{
Daniel Lévêque \\ Université Catholique de l'Ouest - Angers (Francia) \\ 3L.AM-EA4335 \\ daniel.leveque@uco.fr
}

\begin{abstract}
RÉSUMÉ: La traduction en espagnol du verbe «être» français a toujours présenté des difficultés et des doutes à tous les niveaux d'apprentissage et d'étude de la langue de Cervantès, puisque à un signifiant unique français correspondent deux signifiants espagnols. Outre les aspects purement grammaticaux et sémantiques qu'il faudra rappeler (liés à des principes fonctionnels pour les premiers, et polysémiques pour les seconds), il sera intéressant d'aborder la dualité de l'objet d'étude d'un point de vue culturel, voire philosophique, pour ne pas dire métaphysique. C'est ce que nous tenterons de faire en effectuant un parallélisme entre la complémentarité du binôme verbal «ser» et «estar», et la complémentarité du «couple littéraire» emblématique de Don Quichotte et Sancho Panza.

MOTS CLÉS: être, ser / estar, Don Quichotte / Sancho Panza, dualité, complémentarité.
\end{abstract}

\section{UN SOLO SER OS FALTA Y TODO ESTÁ DESIERTO: UNA MIRADA FRANCESA SOBRE LA BIVALENCIA CULTURAL DEL SER EN LA LENGUA ESPAÑOLAA TRAVÉS DE UNA PERCEPCIÓN CERVANTESCA DE LOS VERBOS «SER » $\mathrm{Y}$ « ESTAR »}

\begin{abstract}
RESUMEN: La traducción al español del verbo francés «être» siempre presentó dificultades y dudas en todos los niveles del aprendizaje y estudio de la lengua de Cervantes, pues a un solo significante francés le corresponden dos significantes españoles. Luego de recordar los aspectos meramente gramaticales y semánticos (vinculados a principios funcionales en el caso de los primeros, y polisémicos en el caso de los segundos), será interesante abordar la dualidad del objeto de estudio desde un punto de vista cultural, incluso filosófico, cuando no metafísico. Esto mismo
\end{abstract}


es lo que procuraremos hacer efectuando un paralelismo entre la complementariedad del binomio verbal «ser» $y$ «estar», y la complementariedad de la «pareja literaria» emblemática constituida por Don Quijote y Sancho Panza.

PALABRAS CLAVE: être, ser/estar, Don Quijote/Sancho Panza, dualidad, complementariedad.

Recibido: 26/04/2016. Aceptado: 14/07/2016

\section{En guise d'introduction}

Il est un usage linguistique (ou plus simplement grammatical) qui, à toutes les étapes de l'étude de la langue espagnole, oppose une difficulté récurrente aux apprenants d'abord, puis aux utilisateurs confirmés, pour ne pas parler des chercheurs : nous avons nommé le binôme verbal « ser-estar » en tant qu'il représente deux signifiants face au signifiant unique français « être " (ce rapport de 'deux pour un' n'est pas tout à fait exact d'ailleurs comme on le verra plus loin).

Pour mener à bien cette analyse qui - on va le voir aussi - dépasse la simple prise en compte des aspects linguistiques pour embrasser les domaines culturel, philosophique, voire métaphysique sur fond de littérature classique (en l'occurrence le Quichotte de Miguel de Cervantès), nous avons notamment convoqué - outre des spécialistes des interconnexions entre les langues française et espagnole (comme l'Espagnol Jorge Juan Vega y Vega) -, des critiques littéraires de sensibilité linguistique (comme le Belge Jacques De Bruyne) et des critiques littéraires de sensibilité socio-politique et philosophique (comme l’Espagnol José Ortega y Gasset ou le Mexicain Octavio Paz). C'est ce dernier qui va nous permettre d'amorcer notre réflexion, partant de sa conception de l'Histoire, et, plus particulièrement, de l'histoire mexicaine vue de l'intérieur, sous la perspective de ce qu'on dénomme aussi l'infra-histoire ('intrahistoria'). L'universitaire français Claude Fell rappelait à l'auteur - lors d'un entretien publié en 1975 à l'occasion du $25^{\text {ème }}$ anniversaire de la parution du Labyrinthe de la solitude (El laberinto de la soledad, 1950) - que dans les premières lignes de son essai intitulé Postdata (1970) on peut lire ceci : El mexicano no está en la historia, es la historia (en français : "Le Mexicain n'est pas dans l'histoire, il est l'histoire »), et lui demandait de s'expliquer à ce sujet ; ce que fit Octavio Paz - alors futur Prix Nobel de littérature - non sans omettre d'indiquer, pour commencer, que « l'espagnol a un avantage un peu déloyal sur le français [ce sont ses propres termes] : nous avons estar et ser ». Puis il poursuivait, adaptant cette appréciation linguistique à la question historique : 
'Être [estar] dans l'histoire' signifie être [estar] entouré par les circonstances historiques ; 'être [ser] l'histoire' signifie qu'on est [ser] soi-même les circonstances historiques, qu'on est [ser] soi-même changeant. C'est-à-dire que l'homme n'est pas seulement [ser] objet ou sujet de l'histoire, mais qu'il est [ser] lui-même l'histoire, qu'il est [ser] les changements. (Fell $2010: 422)^{1}$.

Nous constatons ici qu’Octavio Paz adoptait, à une distance de près de quarante ans et de façon 'intégrative’ pourrait-on dire la fameuse formule « moi et ma circonstance » de José Ortega y Gasset, dont nous reparlerons en fin d’article, et sur laquelle repose la dichotomie sémantique ser/estar. Pour l’heure, feuilletons un instant l'œuvre citée Le labyrinthe de la solitude où, dès les premières pages, l'auteur met en avant le postulat dont le reste de l'ouvrage sera la démonstration. Parlant de l'identité mexicaine, et en conclusion de syllogisme, il écrit : « nous sommes [ser], à dire vrai, différents. Et, à dire vrai, nous sommes [estar] seuls » (Paz 1980 : 18)². Ce qui revient à dire, suivant une vision universaliste de l'Homme, que la différence serait porteuse de solitude, ou, à la manière d'un aphorisme, 'qu'être [ser] différent c'est être [estar] seul' (en espagnol : ser distinto es estar solo) ; ou encore, qu'une certaine nature de l'être/ser peut engendrer une certaine situation de l'être/estar... et que, par conséquent, à une nature identitaire essentielle de l'être/ser (on touche ici l'éthos aristotélicien) peut correspondre une situation apparente circonstancielle de l'être/estar (on touche cette fois le pathos non moins aristotélicien) ${ }^{3}$.

Plus simplement, si j’éprouve quelque intérêt pour Ana, par exemple, et que je veuille en savoir plus sur sa personne, ce ne sera pas la même chose de demander ¿Cómo es Ana? que de demander ¿Cómo está Ana? Dans le premier cas (ser), je souhaite me faire une idée de son physique, ou bien connaître sa personnalité ; dans le second cas (estar), je m’enquiers de savoir si elle va bien, sans plus. La première interrogation (ser) porte sur l'être en tant qu'individu (indivis), la seconde (estar) a trait à l'être en tant qu'il répond à un état (un 'stade') d'évolution ou de transformation'.

1. Notre traduction du texte reproduit dans l'édition de Enrico Mario Santí : "El español tiene una ventaja un poco desleal sobre el francés: tenemos estar y ser. 'Estar en la historia’ significa estar rodeado por las circunstancias históricas; 'ser la historia' significa que uno mismo es las circunstancias históricas, que uno mismo es cambiante. Es decir, que el hombre no solamente es un objeto o un sujeto de la historia, sino que él mismo es la historia, él es los cambios”.

2. Notre traduction de : "Somos, de verdad, distintos. Y, de verdad, estamos solos".

3. Voir infra, note ${ }^{\circ} 8$.

4. Ce sont respectivement les concepts de 'Individuo’ et de ‘Estadio' défendus par Federico Silvagni, et étayés par cet exemple, entre autres illustrations (2013 : 44) ; ce que nous développerons plus bas. 


\section{Approches grammaticale et sémantique}

\subsection{En diachronie et synchronie françaises}

\subsubsection{Point de vue diachronique}

Quand nous écrivions à l'instant que le rapport de deux signifiants espagnols pour un signifiant français n'est pas tout à fait exact, c'est qu'en réalité, en français, deux verbes 'être' ont longtemps coexisté, mais ont unifié leur usage au cours des siècles ; ou, pour être plus précis, ce sont deux formes verbales distinctes anciennes qui ont fusionné pour se confondre dans le verbe 'être' unique que nous connaissons actuellement en français.

Voyons de plus près cet aspect de la question tout en gardant à l'esprit la complémentarité sémantique entre l'essence et la circonstance dont nous déclinerons, par la suite, les valeurs dérivées.

Émile Benveniste (1966), dans Problème de linguistique générale 1, et, avant lui, Roland Barthes (1959), ont disserté sur le verbe 'être' et ses bizarreries morphologiques. On retiendra trois étymons indo-européens (du sanskrit) ayant chacun une charge sémantique propre :

a) La racine as ['être en soi' opposé au 'non-être' ; 'exister' ou 'vivre'], qui a évolué vers es ['se trouver'], pour donner, en latin classique, le verbe

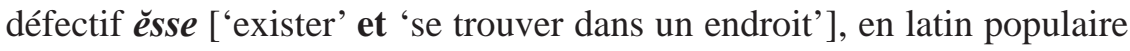

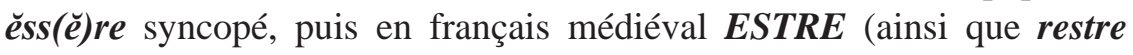
'être de nouveau'). Cette source qui constitue l'axe central débouche sur l'infinitif ÊTRE.

b) La racine sta ['se tenir debout'], qui donnera le verbe latin stare, de même sens ${ }^{5}$, puis le français médiéval $\boldsymbol{E S T E R}$ - prononcé [ester] au XIIème siècle - (ainsi que rester < re stare), et qu'on trouve dans les formes conjuguées du français moderne ÉTAIS, ÉTANT, ÉTÉ (voir l'expression 'ester en justice').

c) La racine bhû/bheu ['être en devenir' ; 'croître', 's'épanouir'], qui a évolué vers les formes latines fui, fuo (infinitif fuere), et qu’on retrouve en français moderne, par exemple au passé simple FUS, FUT, etc.

5. Notons que stare était de sens analogue à celui de ĕsse chez les auteurs latins de l'époque de la décadence (Vème siècle). Cf. Larousse Lexis (1992) ; voir aussi infra la note $n^{\circ} 14$ concernant les verbes espagnols ser et estar. 
Il y eut, à l'origine, coexistence d'emploi à tous les temps de la conjugaison (particulièrement pour esse et fuere), puis utilisation exclusive de tel ou tel verbe pour tel ou tel temps, avant d'aboutir à une unification verbale sur la base de trois paramètres sémantiques dont les deux premiers sont proches, à savoir (Vega y Vega $2011: 30)$ :

a) L'être, le vivant, l'existant (ёsse)

b) Le stable, le demeurant, le durable (stare)

c) Le dynamique, le changeant, l'instable (fuere)

L'unification verbale en question qui s'est opérée entre les formes dérivées

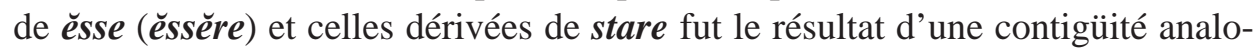
gique influente entre ces formes, tant sous le rapport du signifiant que du signifié6. Il y eut ainsi, au fil des siècles, insertion du verbe de position (stare, ester) au

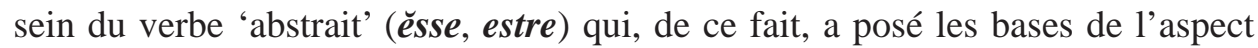
'concret', 'situationnel' du verbe 'être' français, tel que nous le connaissons de nos jours. En d'autres termes, la convergence entre estre et ester s'est soldée par une assimilation syntaxique et sémantique de ester par estre. Dans cette bivalence réside la singularité d'emploi de l'actuel verbe 'être' français'. D’ailleurs, plutôt que de dire que la distinction précédente entre l'énonciation de l'existence (ester) et l'assertion de l'identité (estre) est abolie, selon l'expression forte de Benveniste (1966), il conviendrait d'adopter la position plus nuancée de Vega y Vega (2011) qui indique que si cette distinction n’est plus consciemment établie par les usagers francophones, elle demeure néanmoins détectable par l'analyse : « Être (de estre) ou être (de ester), telle est la question », en somme.

Victor Cousin écrivait, en 1829, dans son Cours de philosophie, que « le mot être est primitif et tout intellectuel. Je ne sache aucune langue - poursuivait-il - où le mot français être soit exprimé par un mot correspondant qui représente une idée sensible » (300). Cet oxymore 'idée sensible’ signale tout à fait la dichotomie sémantique que nous évoquions à l'instant. Nous pourrions même aller plus loin, en compagnie du linguiste espagnol cité précédemment,

6. La lente cohabitation des deux verbes (ester, estre) remonte certainement à la période prélittéraire du français (et était déjà à l'œuvre en bas latin) (Vega y Vega 2011 : 33).

7. Jorge Juan Vega y Vega parle, à ce propos, d’une « décisive structure polyverbale » (2011:32), et même d’une ‘bi-verbalité consubstantielle au verbe être français’ (2011 : 33). Par emprunt, nous pourrions parler de «plénitude de l'être ». 
et faire ressortir que le seul verbe 'être' français rend parfaitement compte des trois dimensions philosophiques de la rhétorique aristotélicienne ${ }^{8}$ :

a) L'éthos qui va à l'essence des choses (relève de la catégorie de l'identification en général, du stable et du définitif, et sert à établir des caractérisations, classements, catégorisations, jugements de valeur et autres équivalences) ; qui a à voir aussi avec le caractère ou l'opinion du locuteur.

b) Le pathos qui exprime l'existence, une circonstance, une vicissitude donnée, une situation; qui réside donc dans le passager, le modifiable ; qui a à voir aussi avec l'effet de persuasion sur l'allocutaire (lecteur ou auditeur).

c) Et le pragma, le logos, en tant que verbe ‘auxiliaire’ qui permet l'agencement des actions (par le biais de constructions passives, impersonnelles, etc.) ; qui a à voir aussi avec le discours rationnel.

\subsubsection{Point de vue synchronique}

Sur le fondement de ces trois dimensions, la valeur sémantique du verbe 'être' français sera dépendante de la fonction syntaxique de celui-ci, et ira decrescendo depuis sa fonction prédicative jusqu’à sa fonction auxiliaire, en passant par sa fonction attributive ${ }^{9}$. Ainsi, on aura, par exemple - et dans l'ordre -, la construction prédicative absolue de Descartes « Je pense, donc je suis » où le verbe 'être' peut se prévaloir de son sens plein d' 'exister' (nous parlerons ici d' 'existentiel absolu') ; la construction attributive de type locatif à la manière de « Je suis en France » (pour laquelle nous parlerons d' 'existentiel relatif') - et ses dérivés traduisant la notion d’état transitoire comme dans « Je suis en colère ${ }^{10}-$, ou bien celle, toujours attributive, de type copulatif où 'être' fait office d'opérateur logique (notamment de marqueur d'équation) comme dans « Je suis intelligent = je suis un 'être' doué d'intelligence », construction plus proche,

8. Citant Aristote, Descartes, Heidegger et Sartre, Jorge Juan Vega y Vega souligne que l'histoire de l'Occident a été marquée par un profond questionnement sur la réalité, l'essence ou la contingence de être (2006 : 948).

9. Cette volatilité sémantique du verbe être est très bien explicitée par Jorge Juan Vega y Vega (2011 : 182). L’auteur tente même d'établir une relation de proportion inversée entre la charge sémantique du verbe être et la longueur des énoncés dans lesquels il apparaît (l'emploi 'absolu' étant le plus court) (180).

10. Selon cette affirmation de J. J. Vega y Vega « qu’un ‘état’ est en fait un type très spécial d’ ‘endroit’ », c’est « se trouver à un moment du temps » (2011 : 199). 
dans ce cas, de certains usages auxiliaires, plus abstraite, où le verbe 'être' est donc aussi moins chargé sémantiquement ${ }^{11}$; enfin, l’abondant usage auxiliaire, justement, comme dans les constructions passives d'action ${ }^{12}$, du style « Je suis rattrapé par la science ", « Je suis ébloui par la lumière » où, sous la prégnance de la fonctionnalité syntaxique, le sens du verbe 'être' se trouve complètement dilué, quoique non totalement évacué13.

Par conséquent, dans le verbe 'être' français « s’amalgament - selon l'expression de Vega y Vega - plusieurs origines morphologiques, d'innombrables constructions syntaxiques, et de nombreuses valeurs sémantiques » (2011 : 240), de telle sorte qu'on peut dire que sa valeur prédicative correspondrait à l' 'être' de estre, sa valeur attributive de type locatif, à l' 'être' ou l' 'y être' de ester (voir l'anglais 'to stay'), celle de type copulatif, à l' 'être' de estre, de même que sa fonction d'auxiliaire d'action. C’est cette multiplicité sémantique - articulée autour de deux axes -, cette bivalence d'ensemble, intrinsèque au verbe 'être' français que la langue espagnole systématisera grâce à la préservation de l'usage de deux verbes distincts $\boldsymbol{S E R}$ et $\boldsymbol{E S T A R}$, deux verbes distincts, certes, mais non dépourvus d'interactions, comme nous le montrerons par des exemples de double construction.

\subsection{En diachronie et synchronie espagnoles}

\subsubsection{Point de vue diachronique}

Nous repartons des racines indo-européennes et latines précédemment détaillées lors de l'étude étymologique du verbe ‘être’ français, mais bifurquons maintenant, par des voies à peine symétriques, vers les destinations morphologiques espagnoles.

11. De l’avis de J. J. Vega y Vega « [...] la transmission de l'identité entre les deux termes reliés par la copule, serait la particularité sémantique la plus définitoire du verbe être en tant que copule, c'est-àdire, en tant qu'opérateur logique » (2011: 205). Voir aussi, sur les différents types de formes copulatives (Vega y Vega 2006 : 955).

12. On pourrait également citer les formes passives d'état, les formes composées des tournures pronominales, les formes composées des verbes intransitifs dits 'de mouvement', ainsi que la périphrase durative « être en train de » suivie de l’infinitif (Vega y Vega $2011: 215$ ).

13. On peut déceler, par exemple, les notions du 'transitoire' ou de l' 'identité' (dans les phrases actives), de la ‘transformation' (dans les constructions passives, pronominales ou intransitives) (Vega y Vega $2011: 223)$. 
C'est ainsi que, d'une part - après un premier temps de confusion phonétique

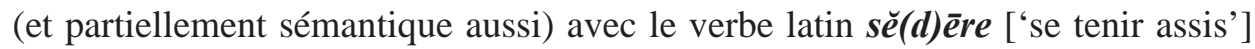
> seer > ser -, le latin populaire $\breve{\boldsymbol{e s s}}(\breve{\boldsymbol{e}})$ re syncopé ['exister'] va susciter majoritairement l'usage du verbe espagnol moderne $\boldsymbol{S E R}$ (dont on trouve la présence dans 'presencia', justement, ou dans 'esencia') (Corominas 1954 : IV 194-195) ; et c'est ainsi aussi que, d'autre part, le latin stare ['se tenir debout'], va se fixer sous la forme espagnole moderne, vocaliquement renforcée, ESTAR, à l'image du français médiéval ESTER. On notera qu'avant d'être sémantiquement démarqués, l'emploi prédicatif du verbe ser et celui du verbe estar furent en concurrence jusqu'à la fin du XVIème siècle (Gili Gaya 1979 : 58-60 ; Lapesa 1981 : 400 § 3) ) $^{14}$, c'est-à-dire - soulignons cette coïncidence pour nous digne d'intérêt - à l'époque même, sinon de la publication, du moins de la rédaction de la première partie du Quichotte de Cervantès (1604), période donc, à partir de laquelle le marquage de la partition de la personnalité humaine se ferait plus net. Enfin, comme dans le cas du français, la forme latine $\mathbf{f u}$ - va générer les flexions verbales de certains temps du passé, telles que FUI, FUISTE, etc. Et, comme aussi pour le français plus haut, la question est posée de savoir quel bon usage faire, en espagnol, des verbes ser et estar : "Ser o estar, he ahí el problema ».

\subsubsection{Point de vue synchronique}

Dans son ouvrage didactique de 2013, intitulé justement ¿Ser o estar? Un modelo didáctico, Federico Silvagni commence par reprendre les lignes de force qui partagent d'ordinaire l'emploi de ces deux verbes espagnol, et tel que cela est enseigné aux non-natifs : ce sont, pour le verbe $\boldsymbol{S} \boldsymbol{E} \boldsymbol{R}$, les notions de 'qualités permanentes' du sujet, d' 'atemporalité' (ou imperfectivité verbale), de 'norme générale', et, à l'inverse, pour le verbe $\boldsymbol{E S T A R}$, les notions de 'caractéristiques transitoires' du sujet, d' 'état résultant' (ou perfectivité verbale), de 'norme individuelle’ - en lien (nexo) avec le circonstanciel - (13-18). Mais là n’est pas l'originalité de son étude. En regard de ces critères traditionnellement exposés aux apprenants de la langue espagnole - critères qu'il juge partiels et donc peu satisfaisants, surtout le premier qui dresse une opposition quelque peu simpliste

14. La concurrence d'emploi prédicatif des verbes ser et estar avant le Siècle d'Or est encore perceptible dans certains usages archaïsants (par exemple, dans ce passage de la salutation mariale - Ave Maria - « El Señor es contigo » [Le Seigneur est avec Vous]) (De Bruyne 1998 : 627, note n 398). C’est aussi l'époque où le pronom personnel vos est utilisé comme traitement familier, avant sa disparition de la Péninsule Ibérique et son maintien en Amérique. 
entre ce qui est permanent (ser) et ce qui est transitoire (estar) (15 $^{15}$ l'auteur avance en effet deux concepts généraux en forme de dichotomie aspectuelle que, pour notre part, nous adoptons volontiers et que nous compléterons par la suite. Il s’agit, d'un côté, des 'prédicats d’individu' (portés par ser), et, de l'autre, des 'prédicats de stade’ (portés par estar) (Silvagni 2013 : 19)16. Globalement, les premiers annoncent les qualités, les caractéristiques intrinsèques et propriétés constitutives du sujet, nous ajouterons : son essence, en quelque sorte sa matière (madera, selon la valeur étymologique de ce mot) ; les seconds renvoient aux situations extrinsèques, aux circonstances accidentelles en tant que causes déterminatives d'un état ; nous avons alors ici affaire à la manière (manera) du sujet, son comportement, ses attitudes, etc. Entrons plus dans le détail ${ }^{17}$.

\subsubsection{Emploi prédicatif comparé de ser et estar}

Dans son emploi prédicatif - intransitif -, le verbe $\boldsymbol{S} \boldsymbol{E}$ a une forte teneur sémantique $^{18}$; il a, de fait, en soi une valeur existentielle que, pour notre part - disions-nous -, nous qualifierons d'absolue : c'est 'exister' (pour une entité) ou 'survenir' (pour un événement). Contentons-nous, pour le moment, de citer un exemple emblématique, à savoir le premier verset du Prologue de l'Évangile de saint Jean qui débute ainsi : « En el principio era [existía] el Verbo... » («Au commencement était le Verbe... ») (Jn. I, 1) [era = imparfait de l'indicatif de ser] ; ou encore l’expression figée « Así sea » («Ainsi soit-il ») [sea = subjonctif présent de ser].

Quant à l'emploi prédicatif - intransitif - du verbe $\boldsymbol{E S T A R}$, qu'on pourrait d'ailleurs assimiler à un emploi attributif, il rend fondamentalement l'idée de situation ou de localisation (dans l'espace ou le temps, ex. : « Je suis ici »), se rapporte à des traits circonstanciels de l' 'être', et a donc toujours en soi une valeur existentielle, certes, mais que nous qualifierons - disions-nous aussi - de relative cette fois. Citons à ce propos la suite du premier verset de saint Jean : « ...y el Verbo estaba con Dios... » (« ...et le Verbe était auprès de Dieu... ») (Jn. I, 1.) [estaba = imparfait de l’indicatif de estar].

15. Voici l'avis de Federico Silvagni sur ce critère d’opposition 'permanent / transitoire' : “[...] es indudablemente el criterio descriptivo menos acertado de toda la tradición gramatical” (2013 : 14, 20 et 33). Ce que nous illustrerons plus bas.

16. 'Predicados de Individuo' et 'Predicados de Estadio'.

17. Rappelons dès maintenant que cette opposition entre 'essence' et 'accidents' fut l'un des objets d’analyse de la philosophie classique dont était imprégné Miguel de Cervantès !

18. Jacques De Bruyne parle, à ce propos, de verbe « sémantiquement plein » (1998: 610). 


\subsubsection{Emploi attributif comparé de ser et estar}

Dans son emploi attributif (de type 'copulatif') - à teneur sémantique neutre, voire insignifiante selon Vega y Vega (1992 : 130) -, le verbe $\boldsymbol{S E R}$, par le lien logique et absolu qu'il établit entre le sujet et l'attribut, induit, d'une part l'idée de caractérisation par l'attribution justement du 'trait distinctif' (notamment dans des expressions descriptives), et, d'autre part, l'idée d'identification (comme c'est le cas, par exemple, dans la construction des définitions ${ }^{19}$ ). Ser permet d'exprimer ce qui définit intrinsèquement le sujet, son essence comme nous l'avons signalé (l'identité - à commencer par le nom de la personne -, la profession ou la fonction, le nombre ou l'équivalence, de même que l'origine, la matière, l'appartenance) ; il cristallise donc ce qui est essentiel, objectif et absolu (De Bruyne 1998 : 611, 622, 624 et 626) ${ }^{20}$. En d'autres termes, ser est un verbe imperfectif [cf. écrire] qui marque une unité indivisible, un 'individu' (individuo), en somme, au sens étymologique où l'entend Silvagni [la rose est une fleur (la rosa es una flor)] (2013 : 24) ; il est, en fin de compte, l'opérateur de la permanence inhérente à l'intemporalité et à l'éternité21. Nous inspirant des développements que faisait Antoine Resano sur la question, nous parlerons ici du verbe ser comme d'un 'opérateur conjonctif' et, symétriquement, du verbe estar comme d'un 'opérateur disjonctif' ainsi que nous allons le voir maintenant ${ }^{22}$.

De son côté, le verbe $\boldsymbol{E S T A R}$ présente également un emploi attributif (de type 'copulatif') et à teneur sémantique neutre, mais à la différence de ser, celuici est tourné vers l'extérieur du sujet - d'où la disjonction tout juste évoquée - ; il établit un lien circonstanciel et transitoire entre le sujet et l'attribut, et dans des expressions descriptives se rapporte aux états dudit sujet (ex. : Je suis en colère) ${ }^{23}$. Estar permet ainsi d'exprimer ce qui marque extrinsèquement le sujet, ses traits accidentels susceptibles de changer au gré des circonstances (le comportement, l'état d'âme, l'attitude physique) ; il renvoie à ce qui est fortuit, subjectif et relatif

19. Ce qui correspond au « fonctionnement copulatif élémentaire » (Vega y Vega 1992 : 130).

20. J. J. Vega y Vega parle de «thématisation », c'est-à-dire de qualité 'consubstantielle’ au sujet, et qualifie le verbe ser employé de la sorte comme « copulatif de l'essence » ou " pragmatique interne » (1992 : 131 et 133).

21. "[...] la atribución con ser tiene ese carácter de definición, de asignación intemporal, definitiva” (Delbarge 2001 : 173).

22. Voir le processus de la 'saisie conjointe' - dans un rapport d'égalité - de Pe (personne externe) et Pi (personne interne) opéré par le verbe ser, et celui de leur 'saisie disjointe' (dans un rapport Pe > et $\mathrm{Pi}<$ ) opéré par le verbe estar, tel que l'expliquait Antoine Resano en 1986 (143 et 146).

23. S'appuyant sur le 'continuum sémantique' entre les deux fonctions (prédicative-locative et copulative) du verbe estar, Federico Silvagni préfère, à raison pensons-nous, parler d'emploi « pseudocopulatif » dans ces cas dérivés, voire figurés (2013 : 31 et 34). 
(De Bruyne 1998 : 615, 622, 624 et 626) ${ }^{24}$. En d'autres termes, estar est un verbe perfectif [cf. écrire une lettre, et, la lettre est écrite] qui atteste le résultat d'une transformation antérieure (un résultat perceptible - constatable - par l'expérience immédiate), qui marque une dualité, un 'stade' (estadio), pour reprendre le terme de Silvagni [la rose est fanée (la rosa está marchita)] ; il est, en fin de compte, l'opérateur du changement inhérent à la temporalité et à la finitude.

\subsubsection{Emploi auxiliaire comparé de ser et estar}

Étant donné qu'il y a « continuité entre la valeur de copule [attributive] et celle d'auxiliaire » (Resano 1986 : 138 et 153), l'opposition susmentionnée, fortement ancrée dans la pensée espagnole, de l’unité (traduite par le verbe ser) et de la dualité (traduite par le verbe estar) se retrouve aussi dans les emplois auxiliaires de ces deux verbes, au sein de structures verbales passives. Dans ce domaine syntactico-logique, on distinguera le 'vrai passif' construit avec $\boldsymbol{S E R}$ dont le sens syntaxique est actif sous l'effet d'un agent - ou 'voix passive d'action' - (ex. : La puerta es abierta por alguien, la porte est ouverte par quelqu'un = on ouvre la porte) du 'faux passif' construit avec ESTAR qui présente en fait un sens syntaxique résultatif - ou 'voix passive d'état' - (ex. : La puerta está abierta, la porte est ouverte) ${ }^{25}$.

De cette première partie linguistique de l'étude il ressort que l'on peut reconnaître assez facilement dans les divers usages des verbes espagnols ser et estar, sinon la morphologie complète, du moins les valeurs sémantiques contenues respectivement dans les anciennes formes françaises estre et ester (mais confondues dans le verbe 'être' du français actuel), valeurs que les hispanophones continuent de distinguer et de dissocier nettement, à savoir en bref, d'une part, ce que nous appellerons ici l'INHÉRENCE, ce qui est 'acquis d'héritage' (rendu par ser), et, de l'autre, l'ADHÉRENCE, le 'rattachement à' (rendu par estar) ${ }^{26}$.

24. J. J. Vega y Vega parle de « rhématisation », c’est-à-dire de qualité substantiellement distincte du sujet, et qualifie le verbe estar employé de la sorte comme " copulatif des circonstances » ou « pragmatique externe » (1992: 133 et 136).

25. On notera que Federico Silvagni considère que la forme progressive espagnole estar + gérondif (qui correspond à la périphrase durative française " être en train de » + infinitif) met en jeu un emploi 'auxiliaire' du verbe estar, ce qui engendre des prédicats de 'stade' (estadio) dynamiques (2013 : 33 et 45).

26. Il est par exemple symptomatique que l'on trouve, en regard, les emplois substantivaux 'el ser humano’ (l’être humain) et ‘el bienestar / malestar humano’ (le bien-être / mal-être [malaise] humain). 


\section{Dimensions péri-linguistiques et méta-linguistiques}

Cela nous amène à aborder maintenant certaines dimensions péri- et métalinguistiques suscitées par les deux facettes de l' 'être' que nous venons de développer ; nous commencerons par fournir quelques interprétations littéraires et culturelles à ce sujet.

\subsection{Interprétations littéraires et culturelles}

Sous ce rapport de l'individu au monde 'circundante' - le monde de la circonstance environnante -, c'est le Quichotte de Cervantès qui a attiré notre attention. Dans les deux facettes grammaticales évoquées précédemment, nous avons vu une 'double face' : celle des deux protagonistes - 'antagonistes' serait plus juste - de l'œuvre citée : Don Quichotte [DQ] et Sancho Panza [SP] qui peuvent personnifier, chacun à sa façon, les deux verbes 'être' commentés.

\subsubsection{Cas simples d'antagonisme}

Il ne faut pas oublier, comme nous l'indiquions plus haut, que Cervantès écrivit son œuvre majeure à une époque où, après une longue période de confusion d'emploi des verbes ser et estar, la pensée espagnole, et sa grammaire qui en systématise l'expression, établissait - rétablissait, devrait-on dire - une nette distinction entre les deux concepts de l' 'être', et, par voie de conséquence, entre les usages verbaux correspondants. Ser et estar entrent ainsi, nous semble-t-il, dans le cadre plus général et traditionnel de la bivalence espagnole, spirituelle et comportementale, que souligne Octavio Paz dans l'essai socio-politico-moral cité en introduction de notre article, cette bivalence qui est «symbolisée - écritil - par l'opposition du réel et de l'idéal » $(1980: 71)^{27}$, et s'est particulièrement manifestée, comme on le sait, dans deux versants de la littérature espagnole : la littérature mystique et la littérature picaresque. On sait aussi que le Quichotte est une œuvre fictionnelle construite en forme de parodie de la littérature chevaleresque antérieure, et sur les agissements de deux 'êtres' : l'ingénieux hidalgo, éponyme du roman, et celui qu’il nommera comme écuyer, Sancho Panza.

27. L'auteur place d'ailleurs cette opposition en contrepoint de la dichotomie entre les concepts de 'fermeture' et d' 'ouverture' qui, de son point de vue, caractérisent la mentalité mexicaine ('chingar' versus 'rajarse'). 


\subsubsection{Don Quichotte}

Alonso Quijano, alias Don Quijote (Don Quichotte), commence précisément, au début du récit (partie I, ch. I), par se dévêtir de son nom propre, de son identité onomastique, pour revêtir l'armure de chevalier errant. Le visage sec et sévère, il n'est plus lui, il est un autre, comme il le dira en franchissant le seuil de la réalité pour entrer dans le monde de son imagination, voire de la folie (avant d'en ressortir d'ailleurs à la fin de l'œuvre ${ }^{28}$ ). D'intellectuel, il se transforme en 'homme d'action' voulant changer les habitudes du monde ; il devient le moteur de l'action romanesque (rappelons-nous, au passage, le sens syntaxique 'actif' du verbe ser). La première série d'épisodes le met en scène dans d'incessantes luttes contre toute forme d'injustice qu'il dédie à Aldonza Lorenzo, alias Dulcinea del Toboso, sa Dulcinée, son idéal amoureux qui est aussi l'une des composantes d'un idéal de vie plus large, transcendantal, et, par là, transformateur, transmutateur de la réalité, pourrait-on dire. " [Don Quichotte] refuse d'accepter le monde réel tel qu'il est, malgré les efforts de conscientisation de la part de Sancho " - écrit Marc Delbarge dans les Actes d'une journée d'étude consacrée à ce sujet en 2005, à Anvers (2007 : 122) ${ }^{29}$-, au point que le monde lui apparaît comme ensorcelé par des esprits mauvais. Pour lui, la réalité environnante, en tant que manifestation permanente de ce sortilège, est donc faussée : elle est l'image inversée de la réalité 'véritable'. Ainsi, des moulins à vent de la Manche sont, pour lui, 'véritablement' des géants changés en moulins (I, VIII), et un troupeau de moutons est, toujours pour lui, 'véritablement' une troupe ennemie changée en troupeau de moutons (I, XVIII). Si l'on reprend la genèse de ces deux exemples connus, nous voyons que l'auteur procède ici par associations visuelles, métaphorisation de la réalité : partant de la taille des moulins - et de leur gigantisme -, il 'invente' les géants ; de même, partant d'un troupeau de moutons - et du groupe effervescent ainsi constitué -, il 'invente' la troupe où l'armée ennemie. Pour le dire autrement, Cervantès dote Don Quichotte de la vision de l'essence des choses, de leur 'être' au sens de $\boldsymbol{S E R}$ (ici, ser alto, être grand [les moulins], et ser un grupo, être un groupe [le troupeau]).

28. Ce que Jacques De Bruyne appelle le phénomène de « dé-quichottisation » (des-quijotización) qui accompagnera la 'mort ordinaire' de Don Quichotte redevenu Alonso Quijano (surnommé 'le bon') [Partie II, chapitre LXXIV] (2007: 18).

29. Notre traduction. 


\subsubsection{Sancho Panza}

Pour ce qui est de Sancho Panza dont le nom même évoque ses facultés pantagruéliques (panza, panse), nous avons affaire à un brave homme débonnaire, à la mine joufflue, sans instruction, aux seuls besoins élémentaires - alimentaires -, voire contingents. S'il est vrai qu'il ambitionne - ou rêve plutôt - d'être le gouverneur d'une île fictive, la Ínsula Barataria, il n'en demeure pas moins d'un empirisme inébranlable. Il fait preuve de bon sens, notamment par l'usage - excessif cependant - des formes proverbiales qui sont, dit-on, le fruit de la sagesse populaire ; preuve aussi de réalisme, voire de matérialisme, comme nous l’avons déjà laissé entendre ; lorsqu'il lui faut prendre enfin des décisions concrètes, il ne se fie qu'à son instinct et à l'acuité de ses cinq sens, ce qui, toutefois, ne l'empêche pas d'être victime de sa trop grande crédulité. Pour toutes ces caractéristiques, il n'est pas déraisonnable, pensons-nous, d'assimiler ce personnage au verbe $\boldsymbol{E S T A R}$, avec les nuances que nous ne manquerons pas d'apporter dans le point suivant.

Un fossé entre les deux antagonistes semble donc bien creusé, mettant en regard, en miroir, deux séries de valeurs ; selon Salvador de Madariaga, Don Quichotte symbolise le courage, la foi, l’idéalisme, l'utopie (il ajoute le libéralisme et la 'gauche'), et Sancho Panza incarne - au propre - la pusillanimité, le scepticisme, le réalisme, le sens pratique (il ajoute aussi la 'réaction' et la 'droite') (2005 : 82). Le dernier paragraphe du chapitre XII de la première partie rend bien compte de ce qui fait encore à ce niveau du récit, précisons-le, figure d'antithèse interpersonnelle :

Sancho, qui vouait au diable ce chevrier trop bavard, pressa son maître d'entrer dans la cabane de Pedro. Don Quichotte finit par céder, mais ce fut pour passer le restant de la nuit à penser à sa Dulcinée, à l'instar des soupirants de Marcelle. Quant à Sancho, il s'installa commodément entre Rossinante et son baudet, et il dormit, non comme un amant éconduit, mais comme un homme moulu et brisé. (Cervantès $1997: 117)^{30}$.

Où l'on devine, derrière ces quelques lignes de mise en situation, toute la différence d'emploi du verbe être espagnol entre, d'un côté, " es cansado » (il

30. Texte espagnol dans la coll. Austral, 1970, 65 : "Sancho Panza, que ya daba al diablo el tanto hablar del cabrero, solicitó, por su parte, que su amo se entrase a dormir en la choza de Pedro. Hízolo así, y todo lo más de la noche se le pasó en memorias de su señora Dulcinea, a imitación de los amantes de Marcela. Sancho Panza se acomodó entre Rocinante y su jumento, y durmió, no como enamorado desfavorecido, sino como hombre molido a coces”. 
est fatigant [DQ]), et, de l'autre, « está cansado » (il est fatigué [SP]). Ce double usage du verbe être avec un même adjectif (participial) nous conduit à examiner les deux personnages littéraires choisis en ce qu'ils sont en fait les « composantes d'une personnalité complexe » (Delbarge 2007 : 123) ${ }^{31}$, des êtres qui, loin de constituer - comme on aurait été enclin à le penser - des pôles définitivement opposés tant sous leur aspect physique que sous leur aspect spirituel, vont avoir une influence réciproque qui va nourrir des interférences et peut-être même une symbiose progressive (souvenons-nous du verbe être français). De miroirs en mirages, ces deux personnages vont en arriver, bien souvent (dans la seconde partie de l'œuvre surtout), à confondre leurs pas, de même qu'il advient - en quelques rares circonstances, fort heureusement - des verbes ser et estar.

\subsubsection{Cas complexes de symbiose}

À ce propos, Salvador de Madariaga, dans son Guide du lecteur du Quichotte, use de deux néologismes et parle chronologiquement de « quichottisation » (quijotización) de Sancho Panza, et, à l'inverse, de " sanchification » (sanchificación) de Don Quichotte.

\subsubsection{1. « Quichottisation » de Sancho Panza}

Suivant le processus de « quichottisation », Sancho Panza se prend à imiter son maître, il adopte son attitude paternaliste ainsi que la manière pédante qu'il a de s'exprimer. Son statut évolue : écuyer, il devient associé et demande un salaire fixe ; il s'instruit et donne, par exemple, des cours de langue à son épouse Teresa ; mais, plus important encore, il se berce des mêmes illusions que Don Quichotte (avant de reprendre pied et de redevenir le brave homme réaliste du début). Pour l'heure, Sancho Panza subit de la part de Don Quichotte une espèce d'endoctrinement sous couvert d'enseignement. Citons, pour preuve, le dernier paragraphe du chapitre XLII de la seconde partie cette fois :

Si tu suis ces préceptes et ces règles, Sancho [dit Don Quichotte après les lui avoir édictés], tes jours seront longs, ta renommée éternelle, tes désirs comblés, ton bonheur ineffable. Tu marieras tes enfants comme tu le souhaites ; ils obtiendront des titres pour eux et leur descendance ; tu vivras en paix et béni de tous. Et au terme de ton existence, quand la mort viendra mettre un terme à ta

31. Notre traduction. 
vieillesse sereine, ce sont les tendres et douces mains de tes arrière-petits-enfants qui te fermeront les yeux. Jusqu'à présent, je t’ai donné des instructions pour la sauvegarde de ton âme. Écoute à présent ce que j'ai à te dire pour celle de ton corps [...]. (Cervantès 1997 : 305-306) ${ }^{32}$.

Si nous revenons à la sphère linguistique, nous observons des cas d'emplois où le verbe $\boldsymbol{E S T A R}$ (comme Sancho Panza) adopte la valeur du verbe $\boldsymbol{S E R}$ (de Don Quichotte) en un processus comparable d' 'essencialisation' (esencialización) que, pour céder au plaisir de néologiser, nous pourrions appeler un 'processus de serisation' (serización) de estar. Par exemple, l'adjectif limpio (propre) relevant fondamentalement du domaine de l' 'existentiel relatif', du 'stade' (estadio), comme dans "El piso ya está limpio » (l'appartement est propre maintenant) peut glisser vers un emploi lié au domaine de l' 'existentiel absolu', de l' 'individu' (individuo), comme dans « Sé que Ana es muy limpia » (je sais qu'Ana est [une personne] très propre). Nous avons relevé un exemple littéraire intéressant de ce cas de figure chez Miguel Ángel Asturias, dans Leyendas de Guatemala où l'auteur guatémaltèque écrit : [...] en verdad creían que los que no mueren donde nacen, no son muertos, sino ausentes, doblemente ausentes [...] (1970: 85) (« [...] ils croyaient fermement que ceux qui ne meurent pas où ils naissent, ne sont pas morts, mais absents, doublement absents [...] »). Le fait d' 'être mort' (on s'attendrait à la forme dominante estar muerto ${ }^{33}$ ) porte ici d'avantage sur l'identité ('être un mort, un défunt') que sur l'état résultant de l'action de mourir, faisant en quelque sorte glisser muerto dans la catégorie lexicale des substantifs (à moins qu'il ne s'agisse, ce qui est toutefois peu probable, d'un usage archaïsant - du Siècle d'Or -, comme c'est le cas de la formule mariale El Señor es contigo (« Le Seigneur est avec Vous ») évoquée dans une note antérieure ${ }^{34}$. On remarquera que cette

32. Texte espagnol dans la coll. Austral, 1970, 528 : "Si estos preceptos y estas reglas sigues, Sancho, serán luengos tus días, tu fama será eterna, tus premios colmados, tu felicidad indecible, casarás tus hijos como quisieres, títulos tendrán ellos y tus nietos, vivirás en paz y beneplácito de las gentes, y en los últimos pasos de la vida te alcanzará el de la muerte en vejez suave y madura, y cerrarán tus ojos las tiernas y delicadas manos de tus terceros netezuelos. Esto que hasta aquí te he dicho son documentos que han de adornar tu alma; escucha ahora los que han de servir para adorno del cuerpo”.

33. Où l'on constate, au passage, que le seul critère de 'durabilité' pour définir le choix entre ser et estar est par trop limitatif, et donc que la recommandation de Jean Bouzet (2006 : 250) d'utiliser comme 'test de situation' l'expression « en ce moment », telle que la rappelle J. J. Vega y Vega, s'avère, à notre avis, quelque peu réductrice. De fait, « el estar muerto » est, à n'en pas douter, un état on ne peut plus durable ! (Vega y Vega 1992 : 135, note $\left.n^{\circ} 13\right)$.

34 . Voir supra, la note $\mathrm{n}^{\circ} 14$. 
'essentialisation' de estar n'induit, dans ces derniers exemples, aucun changement sémantique des attributs, ce qui n'est cependant pas toujours le cas ${ }^{35}$.

\subsubsection{2. «Sanchification » de Don Quichotte}

Le phénomène inverse de « sanchification » de Don Quichotte contribue aussi à opérer le rapprochement comportemental et mental entre les deux hommes. Au fil des aventures qui jalonnent l'œuvre, Don Quichotte se montre de plus en plus prudent, mesuré, dans sa confrontation avec la dure réalité, au point de rompre avec ses idéaux chevaleresques. Don Quichotte, qui est celui qui reçoit le plus l'influence de l'autre, décide, par exemple, de se faire berger (au chapitre LXVII de la seconde partie) et finit même par verser dans l'utilisation des proverbes populaires qu’il décriait tant au début : «-Et moi - repartit Sancho -, je vois que vous faites exactement comme la poêle qui dit au chaudron : ôte-toi de là, tu es plus noir que du charbon. Vous me reprenez parce que j'emploie des proverbes, et vous, vous les débitez par paires! » (Cervantès $1997: 495)^{36}$. Écoutons encore Don Quichotte, à l'heure de sa mort, en citer un : «- Messieurs [...], n’allons pas si vite. Le temps et l'usage rendent l'homme sage. J'étais fou, et j'ai recouvré la raison. J'étais don Quichotte de la Manche, et je suis redevenu Alonso Quichano le bon " (Cervantès 1997 : 535) ${ }^{37}$. Après quoi, il mourra sans plus d'illusions folles (está), mais plutôt l'âme apaisée sous l'effet de la sagesse populaire de celui qui est maintenant devenu son ami et qui espère encore voir vivre son 'bon seigneur' bien des années, dans un monde désenchanté cette fois, le monde du réel, mais, lui, se fait bien des illusions (él es) (Cervantès 1997 : 534) ${ }^{38}$.

L'entrecroisement des verbes ser et estar présente un équivalent linguistique de la « sanchification » où $\boldsymbol{S E R}$ (comme Don Quichotte) adopte les valeurs de ESTAR (de Sancho Panza) en un processus de ‘circonstancialisation’ (circuns-

35. Voir, par exemple, estar despierto (être réveillé) > ser despierto (être éveillé / vif) ; estar vivo (être vivant) > ser vivo (être vif) ; estar maduro (être mûr) > ser maduro (être d'âge mûr) ; estar seco (être sec / une substance) > ser seco (être sec / au figuré, ex. : le ton de la voix).

36. Texte espagnol dans la coll. Austral, 1970, 647 : “-Paréceme -respondió Sancho- que vuesa merced es como lo que dicen: 'Dijo la sartén a la caldera: -Quítate allá, ojinegra’: estáme reprehendiendo que no diga yo refranes, y ensártalos vuesa merced de dos en dos”.

37. Texte espagnol dans la coll. Austral, 1970, 671 : “-Señores [...], vámonos poco a poco, pues ya en los nidos de antaño no hay pájaros hogaño. Yo fui loco, y ya soy cuerdo: fui don Quijote de la Mancha, y soy agora, como he dicho, Alonso Quijano el Bueno".

38. Cf. texte espagnol dans la coll. Austral, 1970, 671. Notons aussi que la relation épistolaire entretenue entre nos deux héros (au chapitre LI de la seconde partie) témoigne de cette 'quichottisation / sanchification’ réciproque (cf. texte espagnol dans la coll. Austral, 1970, 571-574). 
tancialización ou accidentalización) que nous pourrions aussi appeler cette fois, comme nous l'avons osé avec serisation (serización), un 'processus d'estarisation' (estarización) de ser - on nous pardonnera ce dernier néologisme. Par exemple, l'adjectif guapo (beau) relevant fondamentalement du domaine de l' 'existentiel absolu' de l' 'individu' (individuo), comme dans "Ana es muy guapa » (Ana est très belle) peut glisser vers un emploi lié au domaine de l' 'existentiel relatif', du 'stade' (estadio), comme dans " iQué guapa está Ana con esa falda! » (Comme Ana est belle avec cette robe !). Nous terminerons par une réplique littéraire qui illustre parfaitement ce glissement, réplique relevée dans le roman La casa verde, de l'écrivain péruvien Mario Vargas Llosa : [...] Estamos felicísimos de verlo aquí [...] (1981 : 403), («[...] Nous sommes très heureux de vous voir ici [...] »). Le fait d' 'être heureux' (on s'attendrait à la forme dominante ser feliz pour rendre la notion de 'bonheur') est placé ici dans un rapport causal, et donc circonstanciel (de 'voir', 'rencontrer'). Comme précédemment, on remarquera que cette 'circonstancialisation' de ser n'induit, dans ces derniers exemples, aucun changement sémantique des attributs, ce qui, là non plus, n'est pas toujours le cas $^{39}$.

Le relativisme linguistique auquel on parvient et dont on ne peut échapper est évidemment lié au perspectivisme littéraire qui, on l'a vu ici, articule des fragments de vision des choses en un équilibre fragile - celui de l'idéalisme désabusé de Cervantès -, et fait dire à José Ortega y Gasset que « [...] Le Quichotte est une œuvre équivoque » (1976: 91 $)^{40}$ où le « couple antagoniste », pour reprendre l'expression de Salvador de Madariaga (2005 : 82), finit par constituer un être unique en tension, l'être humain universel, en somme - corps et âme chevillés -, dont la bivalence, qui s'est toujours fait sentir avec force dans la culture hispanique, est continuellement révélée par l'habitus langagier qu'autorise le binôme verbal ser / estar.

\subsection{Fondements métaphysiques}

Cette distinction permanente entre l'essentiel et l'accidentel manifestée par les verbes ser et estar - qui, du reste, peut paraître plus importante même que l'est

39. Voir, par exemple - à côté de soy (un) camarero (del restaurante $x$ ) (= je suis serveur) et estoy de camarero (en un restaurante) (= je fais (office de) serveur), où le sens du syntagme nominal (camarero) n'est pas modifié -, le changement sémantique subi en revanche par les syntagmes adjectivaux suivants : ser verde (être vert / couleur) > estar verde (être vert / pas mûr) ; ser listo (être intelligent) > estar listo (être prêt (à)).

40. Notre traduction. 
celle du masculin et du féminin dans le nom - transcende la langue parce qu'audelà de l'être, elle détermine la 'raison d'être' et touche au divin. Il s'agit là d'une différence d'ordre métaphysique que seuls les textes sacrés mettent véritablement en évidence, comme nous allons en voir quelques exemples révélateurs ${ }^{41}$.

Dans la Bible en langue espagnole, ser et estar sont les marqueurs linguistiques par excellence de principes universels. Quand Dieu dit à Moïse : « Yo soy el que soy " (Exode 3,14) (en français : " Je suis celui qui est »), Il exprime la plénitude de l'être qui se suffit à lui-même ; le verbe ser traduit ainsi « la réalité première à laquelle participent tous les êtres [los seres] selon leurs diverses modalités existentielles » (Lucas 2000 : 141). Quand en outre, dans un emploi prédicatif, le Christ dit aux Juifs : "En verdad, en verdad os digo que antes que naciera Abraham Yo Soy » (Jn. VIII, 58) (en français : " En vérité, en vérité, je vous le dis, avant qu'Abraham existât [ou fût], Moi, Je Suis »), le recours à un présent de type gnomique ajoute à l'idée de la plénitude de l'être, celle de la pérennité, de l'intemporalité même de l'existence divine, de son éternitét ${ }^{42}$. Quand enfin, en Jean X, 30, nous lisons : «Yo y el Padre somos una sola cosa » (en français : « Moi et le Père nous sommes un »), c'est alors « la transcendance de l'unité sur la dualité et sur toute multiplicité » (Lucas 2000 : 141) qui est exprimée par le verbe ser. Comment ne pas citer à nouveau, pour finir - mais in extenso cette fois - la version espagnole on ne peut plus explicite du premier verset de l’Évangile de saint Jean où s'intercalent manifestement, et de façon condensée, deux notions métaphysiques de l'être (l'Être Suprême, en l'occurrence). Il est écrit en effet : «En el principio era [ser] [ou existía] el Verbo y el Verbo estaba [estar] con Dios y el Verbo era [ser] Dios » (Jn. I, 1). Alors que la structure latine correspondante emploie à trois reprises le verbe esse, sans distinction, et que le français unifie la formulation autour du seul verbe être (sémantiquement pluriel, certes, mais morphologiquement singulier, comme nous l’avons expliqué) : «Au commencement était [estre] le Verbe et le Verbe était [ester] auprès de Dieu et le Verbe était [estre] Dieu », on observe ici que la langue espagnole permet constitutivement de dévoiler toute la distinction métaphysique entre le Père et le Fils (Verbe) qui préfigure la distinction entre Dieu et le Monde : le premier emploi (ser prédicatif) affirme l'éternité de l'Être Suprême, le deuxième (estar attributif-locatif) indique la 'distance' entre

41. C’est l'idée que développe notamment Jean Lucas dans sa thèse de doctorat (2000 : 138). Rappelons seulement l'opposition qui était exprimée en sanskrit - langue sacrée de l'Inde - entre as (être en soi) et bhû (être en devenir), ce que nous avons mentionné en début d'article.

42. On pourra rapprocher cet emploi du plus prosaïque "[...] al fin y al cabo yo soy el que soy yo, y no tú [...]” (je suis moi, et pas toi), relevé chez l'écrivain colombien Gabriel García Márquez, dans son roman El otoño del patriarca (35). 
l'image et son modèle, le troisième (ser attributif-copulatif) agit comme 'opérateur conjonctif' et réaffirme l'unité divine. Nous voyons donc que, sous cet aspect, les verbes ser et estar sont clairement le reflet, aussi, d'une distinction fondamentale d'ordre méta-linguistique ${ }^{43}$.

\section{En guise de conclusion}

Les points d'ancrage littéraires (le Quichotte) et métaphysiques (la Bible) sur lesquels nous nous sommes appuyé pour mener cette étude corroborent la place linguistique de choix occupée par les deux verbes 'être' espagnols, ainsi que le bien-fondé de leur usage distinctif qui n’est ni opposition ni conflit, mais bien complémentarité : Yo soy yo y mi circunstancia [...] (« Je suis moi et ma circonstance $[\ldots] »)$, selon la formule aphoristique célèbre de José Ortega y Gasset dans Meditaciones del Quijote (1914) ; c'est de cette définition écrite aux confins de l'idéalisme et du réalisme dont peut se prévaloir tout 'être' humain. Ce dernier existe donc, comme nous l'avancions, par inhérence (ser), et tire sa véritable capacité de la communication qu'il entretient avec l'univers, de la pleine conscience de ses circonstances, par adhérence (estar) (Ortega y Gasset $1976: 25)^{44}$. Ces deux concepts d'inhérence et d'adhérence que nous suggérons à nouveau, pour conclure, ne peuvent se concevoir que l'un par rapport à l'autre, en interdépendance. L'inhérence est à l’adhérence ce que la profondeur est à la surface : « [...] de la même façon que la profondeur requiert une surface derrière laquelle se cacher - écrit Ortega y Gasset -, la surface ou couverture requiert, pour exister, quelque chose sur quoi elle puisse s'étendre et qu'elle puisse recouvrir » $(47)^{45}$. En fin de compte, si l'on peut, jusqu'à un certain point, délaisser une partie de l' 'être' au profit de l'autre, et, par exemple, dejar de estar para lograr plenamente el ser (cesser d'être pour accéder pleinement à l'être) ${ }^{46}$, il n'empêche que l'existence

43. Ce passage sur le fondement métaphysique de l'opposition ser / estar est inspiré pour une bonne part de la thèse de Jean Lucas (2000 : 141-143).

44. "El hombre rinde el máximum de su capacidad cuando adquiere la plena conciencia de sus circunstancias. Por ellas comunica con el universo”. Nous mesurons ici pleinement la distance avec le biblique « Yo soy el que soy» (Je suis celui qui est).

45. Notre traduction de "[...] de igual suerte que lo profundo necesita una superficie tras de que esconderse, necesita la superficie o sobrehaz, para serlo, de algo sobre que se extienda y que ella tape”.

46. Comme le laissent entendre ces paroles historiques de Simon Bolivar, rapportées par Gabriel García Márquez dans El general en su laberinto : « Il vaut mieux exister (équivalant à ser) que transformer (équivalant à estar) » (" Primero es existir que modificar »). Il s'agissait pour Bolivar, en septembre 1830, d'assurer la réunification de la Grande Colombie - particulièrement contre Francisco de Paola Santander -, avant que de penser à occuper lui-même à nouveau le pouvoir, ou à toute autre réforme (García Márquez 1989 : 205). 
donnée par 'essence' (ser) ne peut que se construire dans la 'contingence' des faits (estar) : se es, pero hay que decidir cómo se quiere estar (on existe, mais il faut décider de la façon dont on veut vivre) ; en d'autres termes, nous dirions que le combat humain est foncièrement, congénitalement, bipolaire : ainsi, el estar libre se conquista, pero el ser libre se cultiva ('devenir libre se conquiert, demeurer libre se cultive', ou, 'les libertés se conquièrent, la Liberté se cultive', c'est-à-dire que 'sans pour autant être toujours en liberté, l'être humain est un être doué de liberté'). La langue de Cervantès a donc, sur la langue de Molière - comme on le voit en maintes occasions -, cet avantage certain (quelque peu déloyal, selon Octavio Paz, répétons-le) de pouvoir parfaitement délimiter, grâce à cet incontestable atout verbal que nous avons présenté en ces lignes, les contours de ce combat à partie double, qui est beaucoup plus qu'un combat contre des moulins à vent ${ }^{47}$. Là où la sagesse établit une distance, la folie l'abolit - comme elle abolit presque tout d'ailleurs -, ainsi la locura lo cura todo casi, y la muerte acaba por remediar el resto siempre, et la mort - comme le fut celle de Don Quichotte, suivie un an plus tard de celle de son géniteur littéraire - finit toujours par remédier au reste : «- Celles [les bêtises] qui m’ont occupé jusqu’ici, répliqua don Quichotte, sont la véritable cause de tous mes malheurs ; mais j'espère que ma mort, avec l'aide de Dieu, les tournera à mon profit » (Cervantès 1997 : 533)

\section{Références}

ASTURIAS, Miguel Ángel, 1970 (1930). Leyendas de Guatemala. Madrid : Salvat ; Alianza. (Biblioteca Básica Salvat de Libros RTV; ${ }^{\circ}{ }^{50}$ ). BOUZET, Jean, 2006 (1936). Grammaire espagnole. Paris : Belin.

CERVANTES SAAVEDRA, Miguel de, 1970 (1605 / 1615). El ingenioso hidalgo Don Quijote de La Mancha. Madrid : Espasa-Calpe. (Austral ; no 150).

CERVANTES SAAVEDRA, Miguel de (1997). L'Ingénieux Hidalgo don Quichotte de la Manche (version française d'Aline Schulman, avec préface de Jean-Claude Chevalier). Paris : Seuil.

47. Dès lors qu'il veut donner dans la concision, le traducteur de telles formulations espagnoles duelles vers le français se voit obligé d'user de stratagèmes et autres subterfuges : substantivation (être / l'être) ; jeu sur les binômes équivalents de ser / estar ('exister/vivre', 'demeurer/devenir', 'singulier/ pluriel'). Bref, quand un seul (verbe) être vous manque... ! (Un solo ser os falta y todo está desierto...).

48. Texte espagnol dans la coll. Austral, 1970, 670 : “-Los [cuentos] de hasta aquí -replicó don Quijote-, que han sido verdaderos en mi daño, los ha de volver mi muerte, con ayuda del cielo, en mi provecho". 
COROMINAS, Joan (1954). Diccionario crítico etimológico de la lengua castellana. Madrid : Gredos.

COUSIN, Victor (1829). Cours de philosophie. Histoire de la philosophie au XVIII'̀me siècle. Tome II. Paris : Pichon et Didier.

DE BRUYNE, Jacques (1998). Grammaire espagnole-grammaire d'usage de l'espagnol moderne (traduction et adaptation sur la base de la $3^{\text {ème édition en }}$ langue néerlandaise par Alberto Barrera-Vidal). Bruxelles : Duculot.

DE BRUYNE, Jacques (2007). «Introducción a la lectura del mejor libro de todos los tiempos ». Tras las huellas de Don Quijote (Actas de la Jornada dedicada a Don Quijote de la Mancha), Anvers : Lessius Hogeschool, 09/12/2005), éd. et trad. de Lieve Behiels, Ministerio de Educación y Ciencia de España, et Lessius Hogeschool (Associatie K.U. Leuven) : 9-22.

DELBARGE, Marc (2001). Morfosintaxis del verbo español. Louvain, Leusden : ACCO.

DELBARGE, Marc (2007). « Quijotización y sanchificación. El Quijote interpretado por Salvador de Madariaga ». Tras las huellas de Don Quijote (Actas de la Jornada dedicada a Don Quijote de la Mancha), Anvers : Lessius Hogeschool, 09/12/2005), éd. et trad. de Lieve Behiels, Ministerio de Educación y Ciencia de España, et Lessius Hogeschool (Associatie K.U. Leuven) : 119-129.

FELL, Claude (1975). « Vuelta a El Laberinto de la soledad » (conversación con Claude Fell). Plural 50 (texte reproduit notamment dans l'édition de Enrico Mario Santí, 2010) Madrid : Cátedra. (Letras Hispánicas ; nº 346): 417-443. GARCÍA MÁRQUEZ, Gabriel, 1981 (1975). El otoño del patriarca. Barcelone : Bruguera. (Libro Amigo).

GARCÍA MÁRQUEZ, Gabriel (1989). El general en su laberinto. Madrid : Mondadori España. (Narrativa).

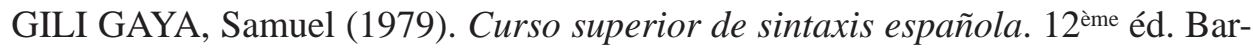
celone : Biblograf. (coll. Vox).

LAPESA, Rafael (1981). Historia de la lengua española (prólogo de Ramón Menéndez Pidal). 9ème éd. Madrid : Gredos. (Biblioteca Románica Hispánica).

LUCAS, Jean (2000). Langues sacrées-Langues profanes. Les traces du sacré dans quelques langues modernes, principalement en espagnol. Thèse de Doctorat. Université de Bretagne Occidentale - Brest.

MADARIAGA, Salvador de, 2005 (1926). Guía del lector del "Quijote". Madrid : Espasa-Calpe.

ORTEGA y GASSET, José, 1976 (1914). Meditaciones del Quijote - ideas sobre

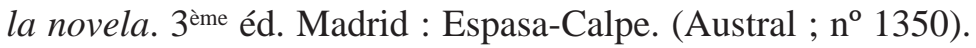


PAZ, Octavio, 1980 (1950). El laberinto de la soledad. Madrid : Fondo de Cultura Económica España. (Colección Popular).

RESANO, Antoine (1986). Étude de systématique espagnole (Acta Hispánica 2). Université de Nantes - Études Hispaniques.

Sainte Bible (La), version française : La Bible de Jérusalem. (2001). Paris : Cerf ; et version espagnole : La Santa Biblia. (1981). Madrid : Ediciones Paulinas.

SILVAGNI, Federico (2013). ¿Ser o estar? Un modelo didáctico. Madrid : Arco/ Libros. (Cuadernos de Didáctica del Español/LE).

VARGAS LLOSA, Mario, 1981 (1966). La casa verde. Barcelone : Argos Vergara. (Libros DB).

VEGA y VEGA, Jorge Juan (1992). "'Ser o no ser'. Un acercamiento pragmático a la utilización de SER / ESTAR en español contemporáneo ». Linguistique hispanique (actualités de la recherche), dir. Gilles Luquet. Limoges : PULIM : 129-138.

VEGA y VEGA, Jorge Juan (2006). " Être, Ser y Estar, Lingüística y ménage à trois ». M. Bruña, La cultura del otro: español en Francia, francés en España. Séville : APFUE-SHF : 948-966.

VEGA y VEGA, Jorge Juan (2011). Qu'est-ce que le verbe 'être' ? (éléments de morphologie, de syntaxe et de sémantique). Paris : Honoré Champion. 


\section{DOCUMENT ANNEXE}

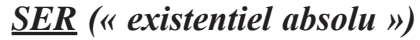

ESSENCE / identité ['éthos']

Intemporel [définitif]

Substantiel [matière]

Nécessaire

Objectif [donnée]

Conjonctif [proximité]

UNITÉ « Individuo » ['indivis']

Singulier

Intangible

Invisible

Dénotatif

Absolu

INHÉRENCE ['acquis d'héritage']

Sens syntaxique actif ['pragma']

Imperfectif $\leftrightarrow$ ESTAR (" existentiel relatif")

$\Leftrightarrow$ CIRCONSTANCE/apparence ['pathos']

$\Leftrightarrow \quad$ Temporel [transitoire]

$\Leftrightarrow \quad$ Accidentel [manière]

$\Leftrightarrow$ Contingent

$\Leftrightarrow \quad$ Subjectif [perception]

$\Leftrightarrow$ Disjonctif [distanciation]

$\Leftrightarrow$ DUALITÉ « Estadio » [stade]

$\Leftrightarrow$ Pluriel

$\Leftrightarrow$ Tangible

$\Leftrightarrow$ Visible

$\Leftrightarrow$ Connotatif

$\Leftrightarrow$ Relatif

$\Leftrightarrow$ ADHÉRENCE ['(r)attachement à']

$\Leftrightarrow \quad$ Sens syntaxique résultatif ['pragma']

$\Leftrightarrow$ Perfectif

$$
\begin{aligned}
& \text { Dualité culturelle espagnole } \\
& \text { Místicos } \Leftrightarrow \text { Pícaros } \\
& \text { Abstrait } \Rightarrow \text { idéalisme } \Leftrightarrow \text { Concret } \Rightarrow \text { réalisme } \\
& \text { Profondeur } \Leftrightarrow \text { Superficialité } \\
& \underline{\text { DON QUICHOTTE }} \leftrightarrow \leftrightarrow \underline{\text { SANCHO PANZA }}
\end{aligned}
$$

SER-ESTAR OU LES DEUX FACETTES DE L'ÊTRE EN LANGUE ESPAGNOLE 